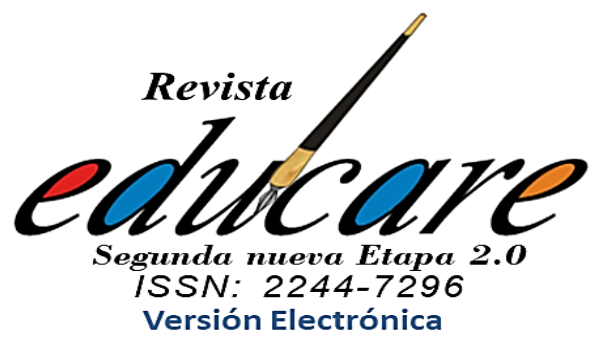

Volumen 25 № 3 Septiembre - Diciembre 2021

$(163-186)$

\title{
EDUCACIÓN Y \\ TRANSGÉNERO: ABORDAJE DE LA REALIDAD DESDE UNA HISTORIA DE VIDA
}

Ecuador

\author{
EDUCATION AND TRANSGENDER: \\ APPROACHING REALITY FROM A LIFE \\ STORY
}

\begin{abstract}
Contacto
*E-mail: natasha21alava@gmail.com

**E-mail: mercedes.cedeno@utm.edu.ec
\end{abstract}




\section{Introducción}

La vulnerabilidad es un concepto dinámico que involucra diversos factores en el que se incluye el contexto, el tiempo y los acontecimientos en la cual las personas pueden ser debilitadas o deterioradas por el impacto de estímulos negativos. Implica ser susceptible de recibir o padecer situaciones que tienden a atenuar a la persona física y emocionalmente. Desde el punto vista etimológico, el termino provine del latín con el prefijo vulnus que significa herida y obilis que indica "se puede" y el sufijo dad que significa cualidad, lo que da entender que la vulnerabilidad es la cualidad para poder ser herido. En palabras de Turner et al. (2003) la vulnerabilidad es definida como inseguridad y sensibilidad del bienestar de las personas en situaciones de transformación, e implícitamente en su respuesta resiliente a los riesgos que se afrontan durante los impactos que se consideren negativos.

En este mismo sentido, la vulnerabilidad no es una particularidad inquebrantable en las personas, así lo refirieren Díaz Barriga et al (2017), sino que está sujeta, al menos parcialmente a factores que pueden cambiarse y transformarse, que permiten la intervención la prevención o la capacidad y mecanismos para superar los efectos de los mismos ante una situación de riesgo, dando cabida a procesos resilientes, siendo estos considerados como mecanismos emocionales que afloran para enfrentar con éxito situaciones adversas.

Partiendo de estas presunciones, Grotberg (2009) señala que el papel de la resiliencia es desarrollar las capacidades humanas de enfrentar, sobreponerse y ser fortalecido, incluso transformado por las experiencias con base a la adversidad, asimismo, permite transitar a sectores personales emocionalmente confortados, a la vez que ayuda a crear estrategias para el afrontamiento a situaciones de vulnerabilidad.

Es decir, la resiliencia en el ser humano está vinculada con la posibilidad de alcanzar un desarrollo psicológico y social saludable y exitoso, a pesar de la vivencia de situaciones que vulneran al individuo (Kotliarenco, Cáceres y Fontecilla, 1997). Lo referido permite inferir, que la vulnerabilidad vivida por las personas puede permitir procesos resilientes, dado que es un factor que se da de forma coyuntural y que puede ser modificado, para Cardoso (2019) la resiliencia es un componente integrante de la vulnerabilidad.

Estos factores de vulnerabilidad, aunque se dan de forma coyuntural como refiere el autor antes citado se ven reflejados en la violación de los principios de los derechos humanos. En este 
sentido los principios sobre la aplicación de la legislación internacional de derechos humanos en relación con la orientación sexual y la identidad de género también conocida como Principios de Yogyakarta (2007) sostiene que la orientación sexual y la identidad de género son esenciales para la dignidad y la humanidad de toda persona y no deben ser motivo de discriminación o abuso.

No obstante, sostienen los mismos principios, que las violaciones a los derechos humanos basadas en la orientación sexual o la identidad de género reales o percibidas por las personas constituyen un patrón global arraigado, que es motivo de seria preocupación. Entre estas violaciones se encuentran los malos tratos, las agresiones sexuales y las violaciones, las injerencias en la privacidad, entre otros, las cuales se ven agravadas por situaciones que tienden a crear vulnerabilidad en estas personas como lo es el odio, las discriminación y exclusión. Aunado a esto la American Psychological Association (APA) (2008) señala que muchas personas transgenero son víctimas de delitos basados en el odio, de discriminación sutil, que va desde miradas de reojo o gestos de desaprobación, o molestia, hasta preguntas agresivas sobre las partes de su cuerpo, es aspectos que conllevan a la persona a un estado de vunerabilidad.

En este mismo sentido, Granado (2020) exponen que las personas transgénero constituyen una minoría expuesta a vulnerabilidades sociales, Educativas, económicas y psicológicas basadas en expresiones abiertas de discriminación, violencia física y emocional, acoso verbal, rechazo y las lleva a experimentar problemas en su personalidad en la que se destaca la depresión, la ideación suicida, las conductas de autolesión y los trastornos alimenticios.

La situación antes planeada no es ajena a la colectividad de personas transgeneros de la provincia de Manabí, en estudio realizado por Gutiérrez et, al. (2017) se encontró entre los aspectos psicosocial, que la realidad social en la que se desenvuelve el grupo es adversa, en la misma, los autores sostienen que la vida de una persona con identidad transgenero en la localidad se dificulta al momento de sentir los improperios de algunos ciudadanos; siendo estos victimas de burlas y epítetos además de sentir el malestar de otras personas al ingresar a sitios públicos, asistir a la escuela entre otros. En términos generales en la ciudad de Portoviejo las personas con identidad transgenero han sentido discriminación siendo violentados sus derechos a la salud, a la educación, a la empleabilidad y libre tránsito social. 
No obstante, hay grupos de jóvenes transgenero en la provincia de Manabí, específicamente en la ciudad de Portoviejo Ecuador que ha encontrado en la resiliencia la esencia para promover su crecimiento y desarrollo personal generando bienestar, autovalía y autoaceptación, retomando los sitios públicos y espacios educativos sin prejuicio alguno. De allí que el siguiente artículo tiene como objetivo dar a conocer los factores educativos, sociales y familiares identificados de vulnerabilidad vividas por estos jóvenes, así como las habilidades resileintes desarrolladas para superar las mencionadas situaciones y retomar una vida normal.

\section{Desarrollo}

\section{Espacios que generan vulnerabilidad}

El concepto de vulnerabilidad con el tiempo se ha disociado del termino de pobreza, teniendo en la actualidad como componte explicativo desde punto vista social tiene una connotación de inseguridad e indefensión que experimentan las personas a consecuencia de un impacto provocado por algún tipo de evento de carácter traumático.

En virtud de la definición antes expuesta, la colectividad transgenero es sometida a situaciones de vulnerabilidad a cada momento dado que ellas tal como lo expone Napiarkorvski (2012) desestabilizan todas las categorías binarias establecidas en la sociedad: varón-mujer, masculino-femenino, sexo-género. Entre las instituciones que generan vulnerabilidad a las personas transgenero se presenta las instituciones educativas, la familia, y la sociedad.

\section{Las instituciones educativas}

De acuerdo al informe de la UNESCO (2019) señala que la violencia homofóbica y transfóbica en la escuela es un problema mundial, el cual tiene un impacto negativo significativo en la educación, las perspectivas de empleo, la salud y el bienestar de los estudiantes. Los estudiantes víctimas de violencia homófoba y transfóbica por lo general presentar un estado de salud física y mental más débil que el promedio, con mayor riesgo de ansiedad, miedo, depresión, autolesiones e incluso suicidio. De allí que la UNESCO recomienda que la respuesta del sector educativo sea respetuosa con los derechos, esté centrada en el alumno y sea inclusiva y participativa.

En este mismo sentido, en un estudio realizado por la Federación Estatal de Lesbianas, Gais, Transexuales y Bisexuales (FELGTB) señala que el 58\% de los alumnos transgenero sufren discriminación en la escuela, las víctimas aseguran que el centro educativo no tomó 
ninguna medida para castigar a los victimarios, exponen además que 'Las generaciones de estos jóvenes tienen un miedo que los paraliza porque los institutos educativos te invitan a irte en vez de ayudarte". Para Rosero (2017) es importante que las instituciones educativas se encuentren preparadas para asesorar a los padres de jóvenes transgenero, como también, que trabajen en conjunto para que exista un ambiente educativo oportuno y adecuado para el individuo.

\section{La familia}

La familia es el espacio natural donde se fundamentan los valores, normas y costumbres que determinan las interacciones y comportamientos sociales (Fernández, 2007, p.33). En tal sentido, el núcleo familiar se convierte en el primer escenario donde el individuo desarrolla y experimenta emociones a través de la interacción con otras personas y con la sociedad. Por consiguiente, es el contexto familiar donde se determinan las pautas y dinámicas de socialización, y se estructuran las bases normativas de la conducta social, las relaciones interpersonales las cuales determinan el desarrollo psicológico y social del ser humano y los fundamentos de su identidad sexual y su rol de género (Fernández, 2007).

No obstante, cuando en el núcleo familia no se comprende una conducta y se ignora por completo se produce un fenómeno que, de acuerdo a lo planteado por Alarcón de Soler, (2006) es un proceso de exclusión que posiblemente influye para que se convierta en un secreto, ya que los miembros de la familia tienen sentimientos de vergüenza, ante la imagen social que desean mostrar. En este sentido la opinión o el concepto que la familia tenga sobre uno de los miembros va a perjudicar o favorecer el crecimiento y desarrollo en toda su estructura tanto de personalidad como de las decisiones que vaya a tomar, o la confianza que vaya a sentir para enfrentarse con el entorno.

\section{La sociedad}

El fenómeno del transgénero y la transexualidad ha existido siempre y se ha dado en todas las culturas. Sin embargo, en las últimas décadas se ha hecho más visible socialmente. Las personas transgénero desean reconocerse a sí mismas y ser aceptadas por su verdadera identidad de género y desarrollarse socialmente. Para ello padece numerarme sufrimiento personal que se ve agravado por la incomprensión, e incluso el rechazo y la discriminación de la sociedad. 
Es de hacer notar que dentro del imaginario social las personas transgenero no figura ocupando empleos estables y con salarios dignos o teniendo acceso a estudios universitarios. Al contrario, y específicamente las mujeres por lo general están destinadas al trabajo sexual, o dedicarse al estilismo, y todo ello se debe porque a nivel social existe para estas personas discriminación laboral. Señala Borella et al (2020) que el acceso a derechos básicos como la educación, la salud y el trabajo aparecen denegados o en condiciones precarizadas a las personas transgénero.

\section{Factores de resiliencia}

La fuente de interactividad de la resiliencia tiene su base en la forma en como el sujeto se apropia de la realidad a través del habla y esta abre la posibilidad al comportamiento saludable o de superación de los eventos traumáticos (Saavedra y Villalta 2008). En tal sentido refieren los autores citados que la resiliencia es un rasgo personal que se cultiva a lo largo de la historia de sujeto, y que posiblemente, se constituye como vinculo temprano siendo condición para el desarrollo de una particular apropiación de los sucesos de la vida. De allí que para que el sujeto se apropie de manera resiliente de la realidad Grotberg (citado en Saavedra y Villalta 2008), propone una clasificación de 3 categorías:

1. aquellas que tienen que ver con el apoyo que la persona cree que puede recibir (yo tengo...).

2. aquellas que tienen que ver con las fortalezas intrapsíquicas y condiciones internas de la persona (yo soy..., yo estoy) y

3. aquellas que tienen que ver con las habilidades de la persona para relacionarse $y$ resolver problemas (yo puedo...).

Por su parte, Saavedra (2008) desarrolla un modelo emergente del estudio de casos donde señala que:

1. la Respuesta resiliente es una acción orientada a metas, respuesta sustentada o vinculada

2. una Visión abordable del problema; como conducta recurrente

3. Visión de sí mismo, caracterizada por elementos afectivos y cognitivos positivos o proactivos ante los problemas; los cuales tienen como condición histórico estructural y 
4. Condiciones de Base, es decir un sistema de creencias y vínculos sociales que impregnan la memoria de seguridad básica y que de modo recursivo interpreta la acción específica y los resultados. A partir de este modelo, la autora reúne el carácter histórico de cómo se constituye la resiliencia proyectando sus posibilidades más allá de la acción y sus resultados para postular la reorganización de los diversos factores que describen los diversos estudios, en ámbitos de menor a mayor profundidad en la reflexión de la acción, (Saavedra y Villalta 2008).

De allí que, al cruzar las dos miradas teóricas, las "dimensiones del yo" de la Grotberg la respuesta Resiliente de Saavedra se llega a constituir 12 factores que describen distintas modalidades de interacción del sujeto consigo mismo, con los otros, con sus posibilidades tal como se muestra en el siguiente cuadro

\begin{tabular}{l|l|l|l|l|l|l|l|l}
\hline & \multicolumn{7}{|c}{ Niveles de estructuración } \\
\hline $\begin{array}{l}\text { Modalidad de } \\
\text { interación }\end{array}$ & \multicolumn{2}{|l|}{$\begin{array}{l}\text { Condiciones de } \\
\text { base }\end{array}$} & \multicolumn{2}{l}{$\begin{array}{l}\text { Visión de si } \\
\text { mismo }\end{array}$} & \multicolumn{2}{l}{ Visión de problema } & Respuestas resilientes \\
\hline Y soy yo estoy & F1 & Identidad & F2 & Autoestima & F3 & Satisfacción & F4 & Pragmatismo \\
\hline Yo tengo & F5 & Vinculo & F6 & Redes & F7 & Modelo & F8 & Metas \\
\hline Yo puedo & F9 & Afectividad & F10 & Autoeficacia & F11 & Aprendizaje & F12 & Generatividad \\
\hline
\end{tabular}

Factores de resiliencia escala SV-RES (Saavedra y Villalta, 2008)

\section{Metodología}

El estudio se enmarca en una perspectiva cualitativa, a través de un estudio de caso, el cual en palabras de Piñero et. al (2019) esta metodología permite el abordaje de un caso o fenómeno actual en su auténtico contexto. Específicamente los autores refieren "caso" "aquellas situaciones o entidades sociales únicas que merecen interés de investigación” (p, 128). Igualmente, Cifuente (2011) describe que el estudio de caso es una alternativa que permite conocer situaciones problémicas al tiempo que se orienta a comprender dinámicas sociales particulares que están relacionadas con factores de riesgo, o su acercamiento puede ofrecer alternativas de transformación social. De allí que los estudios de caso representan una descripción holística e intensiva que analizan una instancia única un fenómeno o una unidad social (Piñero et al, 2019), como lo es en este caso los jóvenes transgenero de la ciudad Portoviejo. 
En lo que respecta la perspectiva cualitativa, se asume dado que permite recuperar las experiencias fenomenológicas de las personas transgero y entender su papel como sujetos sociales en un contexto de vulnerabilidad y resiliencia. En palabras de Ravelo, (2005) (Citado por Díaz Barriga et, al. 2017) investigar estos hechos lleva implícito toda una carga emocional y subjetiva. Es por ello que las teorías, los datos y los conceptos de la ciencia tradicional positivista no funcionan, para entender el fenómeno.

En lo que respecta a los actores sociales, en el estudio participaron 5 personas con edades entre los 18 y 23 años de edad transgenero miembros de la provincia de Manabí de la ciudad de Portoviejo los cuales accedieron a aportar a través de narraciones, escritos autobiográficos y entrevistas a profundidad a expresar a viva voz sus vivencias y resignificar sus experiencias en torno a los factores de vulnerabilidad y las habilidades resilientes desarrolladas.

La entrevista a profundidad de acuerdo a Taylor y Bogdan, (1992) hace referencia a los reiterados encuentros cara a cara entre el investigador y los informantes, encuentros que fueron dirigidos hacia la comprensión de las perspectivas que tienen las personas enfocas en las categorías de vulnerabilidad y resiliencia. Es de acotar que la entrevistas para conocer los aspectos de resiliencia de los actores sociales fue orientada con base a los 12 factores específicos de resiliencia, que propone Saavedra y Villalta, (2008). Mientras que los aspectos de vulnerabilidad estuvieron dirigidos en torno a los factores Sociales, Educativos y familiares.

Una vez realizada la recolección de la información, y la correspondiente organización y transcripción en los protocolos debidamente identificados, devino uno de los momentos más importante en el proceso de investigación en la perspectiva metodológica cualitativa, la cual consistió en el análisis comprensivo, interpretativo. De esta manera que durante el análisis de la información se siguió los procesos propuestos por Strauss y Corbin (2002 el cual se basa en: (a) descripción, (b) ordenamiento conceptual para realizar la interpretación de los hallazgos.

Para cerrar este apartado es preciso destacar que a partir del transitar filosófico y metodológico, que tejen los hilos de la investigación, es insoslayable hacer referencia que la génesis de las investigaciones cualitativas radica en la subjetividad en el marco de la comprensión de la realidad humana, por lo que es imperativo aceptar varios enfoques de la misma, es decir, que la cosmovisión propia de cada investigador se visualiza desde diferentes aristas de la realidad existente. De modo que la legitimidad en este tipo de investigaciones según González (2006) es: "un proceso intrínseco al propio desarrollo de la investigación, que se define 
por la apertura y seguimiento de nuevas zonas de sentido que amplían de forma progresiva la significación del modelo en construcción” (p. 82).

En este contexto se legitima el estudio dado que su eje temático está dentro de los lineamientos de las ciencias sociales lo cual permite que se suscribe a la sublinea de investigación resiliencia e intervención social. Es decir que esta investigación es un aporte para enriquecer la acción de la línea de investigación, además que servirá de referencia para futuras investigaciones en esta área del saber.

\section{Resultado del estudio}

Este proceso de develar los resultados tiene sus bases en la sistematización de la información lo cual condujo a una reducción o codificación axial que permitió conocer, analizar, interpretar y reflexionar, para mirar y acercarse al mundo subjetivo de las personas y dar a conocer los conceptos emergentes. Partiendo de esta premisa, los 5 actores sociales que participaron en el estudio hicieron una narrativa de vida en la que destacan sus vivencias en su estado de vulnerabilidad, en la mismas todos coinciden que estas inician en el contexto escolar específicamente en la adolescencia y se extendieron hasta la adultez. Posteriormente exponen sus experiencias de cómo fue su transitar al proceso de resiliencia, información que fue se genera a partir de los factores de resiliencia en la modalidad de interacción, "yo soy" "yo estoy" "yo tengo" "yo puedo".

\section{Conceptos generados en los factores de vulnerabilidad}

\section{Primer concepto Emergente: La Sociedad}

La no aceptación social de los jóvenes transgero se traduce en la mayoría de los casos en transfobia, lo cual implica miedo, odio, desconfianza, incomodidad o no aceptación de las personas. Las situaciones de violencia vividas por los actores sociales se basan en este concepto, los cuales están dentro de los factores sociales, educativos y psicológicos, haciéndolos sentir en un estado de desvaloración, baja autoestima, aspectos que marcaron fuertemente sus existencias. En tal sentido, se muestra a continuación con bases a las narrativas de los actores sociales la reducción axial, en este particular las relacionadas con los de factores sociales: 


\section{Cuadro 1}

Acciones de violencia dentro del factor social

\begin{tabular}{|c|c|c|c|c|c|c|}
\hline \multicolumn{6}{|c|}{ Factores sociales } & \\
\hline & \multicolumn{5}{|c|}{ Acciones } & \\
\hline Actor social & Burlas & Rechazo & discriminación & Improperios & $\begin{array}{c}\text { Agresiones } \\
\text { físicas }\end{array}$ & $\begin{array}{c}\text { Agresiones } \\
\text { Verbales }\end{array}$ \\
\hline \multicolumn{7}{|l|}{ AS1 } \\
\hline \multicolumn{7}{|l|}{$\overline{\mathrm{AS} 2}$} \\
\hline \multicolumn{7}{|l|}{ AS3 } \\
\hline \multicolumn{7}{|l|}{ AS4 } \\
\hline AS5 & & & & & & \\
\hline
\end{tabular}

Las acciones antes expuestas generaron de acuerdo a los expresado por los actores sociales un sentimiento de inferioridad, con poca o nula cavidad en el contexto donde habitan, sentir el desprecio humano por otro humano por el solo hecho de vivir una sexualidad diferente los lleva a experienciar situaciones de hostilidad con tanta frecuencia y que no ha sido fácil de internalizar esos sentimientos de rechazo. Por otro lado, sentirse agredido por la burla es otro aspecto que lesiona profundamente la autoestima.

Para mí era muy fuerte salir a la calle.... sentía las miradas de las personas y escuchaba sus burlas porque mis movimientos corporales, y mi tono de voz no era como los de otros niños de mi misma edad (yo tenía 14 años) ... con mucha frecuencia me gritaban cosas que me hacían sentir muy mal conmigo mismo y en ese momento muy poco entendía lo que me estaba sucediendo. (Actor Social 3).

Las necesidades de las personas transgénero son poco comprendidas, por la sociedad y por el área de la salud. La carencia de información apropiada, genera estigma y prejuicio, que conduce a la discriminación, el acoso y el abuso, generando deterioro en la salud emocional de los individuos transgénero, (Ruiz Cortes, 2017)

Una vez sufrí un accidente, y me llevaron al centro médico, en el rostro de la enfermera que me estaba curando se le notaba su rechazo hacia a mi... prácticamente no me quería tocar (Actor Social 1).

Las personas que perciben rechazo a lo largo de sus vidas tienden a desarrollar sentimientos de baja autoestima y bajo sentido de competencia o autoeficacia, estas emociones 
que se generan al percibir el rechazo inducen a las personas a desarrollar una visión negativa del mundo (Rohner y Carrasco, Miguel 2014).

Una vez tomé un transporte público, y todos empezaron a burlarse de mí, fue terrible sentir el rechazo de las personas, empezaron a gritarme cosas y el chofer me dijo que me bajara de su carro porque él no aceptaba a personas como yo (Actor Social 5)

\section{Segundo Concepto emergente: La familia}

La familia además de ser la responsable de los cimientos y valores que se instauran en las personas también se convierte en una red de apoyo para sus miembros cuando se necesite. Sin embargo, en el caso de aceptación de un miembro transgero son muy pocos las familias que viven un proceso de aceptación sin trauma, y esto por general se debe a que a que tradicionalmente la familia es un núcleo biparental; que responde a un paradigma social patriarcal que establece como construcción identitaria la heterosexualidad. Y el hecho de que uno de sus miembros este dentro de una situación transgénero genera en ella un escenario de disrupción afectando de manera directa el vínculo y la comunicación familiar, además que causa trauma, rechazo a la persona afectada. En virtud de estas apreciaciones, las vivencias de los actores sociales se centraron en las siguientes acciones:

\section{Cuadro 2}

Acciones de violencia dentro del factor Familiar

\begin{tabular}{|c|c|c|c|c|c|c|}
\hline \multicolumn{5}{|c|}{ Factores Familiar } & \\
\hline & \multicolumn{5}{|c|}{ Acciones } & \\
\hline $\begin{array}{c}\text { Actor } \\
\text { social }\end{array}$ & $\begin{array}{c}\text { No me } \\
\text { aceptaron }\end{array}$ & $\begin{array}{c}\text { Me botaron de la } \\
\text { casa }\end{array}$ & $\begin{array}{c}\text { Agresión } \\
\text { física }\end{array}$ & $\begin{array}{c}\text { Agresión } \\
\text { verbal }\end{array}$ & Aislamiento & Vergüenza \\
\hline AS1 & & & & & & \\
\hline AS2 & & & & & & \\
\hline AS3 & & & & & & \\
\hline AS4 & & & & & & \\
\hline AS5 & & & & & & \\
\hline
\end{tabular}

La mayoría de estos actores sociales manifestaron, el estado de vulnerabilidad que vivieron en sus hogares al develar su identidad transgénero, para algunos de ellos quizás no esperaban acciones de rechazo de crítica y de agresión. Para Perezi, (2016) el apoyo familiar 
como agente socializador es fundamental en la formación de la personalidad y de las construcciones y prácticas sociales que permiten que los individuos comprendan, se adapten e interactúen de acuerdo a las normatividades sociales, no obstante, para los algunos entornos familiares no es fácil asimilar y aceptar una situación que este fuera de los que dictan los parámetros sociales, culturales y religiosos.

Cuando le conté a mi mamá lo que me estaba sucediendo se molestó mucho conmigo, incluso me dio muchas cachetadas y me dijo que sería la vergüenza para toda la familia, me dijo que era mejor que me enderezara o asumiera las consecuencias. Con el tiempo terminó botándome de la casa y me quemó toda mi ropa, fue muy triste lo vivido ese día, por mi mente paso por un momento el pensamiento de quitarme la vida. el miedo y la depresión se apoderaron de mi al sentirme solo. (Actor Social 4)

Para Hoffman, (2014) las familias de individuos transgénero, tienden a desarrollar actitudes negativas hacia ellos, según las cuales pueden llegar incluso a desconocerlos como parte de su sistema familiar.

(...) El día en que mis padres se enteraron, yo entre molesto a casa porque en la calle se habían burlado de mí, mi mamá se preocupó al ver el estado en que había llegado y me vi obligado a contarle lo que me estaba sucediendo, yo esperaba que ella me comprendiera, pero no fue así, se lo contó a mi papá y el me dio una paliza muy fuerte, ese día me quise morir, sentí desprecio por mí mismo, quería salir corriendo sin rumbo .... como ya era mayor de edad me fui a casa de una amiga ellos me dijeron que no querían saber de mí. (Actor Social 2)

Por su parte Budge et al. (2013) afirman que, los individuos transgénero reciben poco apoyo de sus familiares y esta situación de rechazo genera un impacto negativo en su forma de vida.

Yo le conté a mi hermano mayor lo que me estaba sucediendo, él me dijo que se avergonzaba de mí y que no quería que lo vieran conmigo, yo me aislé mucho, me la pasaba encerrado en mi cuarto, a veces pensaba en quitarme la vida y terminar con todo... lo que más me atormentaba y me hacía sentir miedo era el hecho de pensar que si mi mamá se enteraba iba a sufrir mucho, y de hecho así fue... yo intenté suicidarme.... (Actor Social 2)

la familia cumple con una función determinante en el desarrollo psicosocial de los individuos donde se construyen las bases normativas para la interacción de los diferentes 
contextos sociales. Sin embargo, los estudios en personas transgénero muestran que la falta de apoyo familiar puede generar problemas psicológicos incrementando la vulnerabilidad y propensión a tener conductas riesgosas.

\section{Tercer concepto emergente: Contexto educativo}

Las instituciones educativas, forman parte de los contextos sociales con mayor discriminación y violencia que marca de manera emocional a las personas transgénero, se convierte en otro eslabón de esa cadena de burla, rechazo y marginalidad. En este sentido Carvaja (2018) señala que, con frecuencia, en las escuelas y colegios, independientemente de que las personas homosexuales o transexuales sean visibles o no, se hace patente la transfobia y la homofobia por medio del lenguaje, los chistes o el uso de términos que pueden considerarse ofensivos e insultos.

\section{Cuadro 3}

Acciones de violencia dentro del factor Educativo

\begin{tabular}{c|c|c|c|c|c}
\hline \multicolumn{5}{c}{ Factores Educativo } \\
\hline & \multicolumn{5}{c}{ Acciones } \\
\hline Actor social & Burlas & Exclusión & Agresión física & Rechazo & Deserción \\
\hline AS1 & & & & & \\
\hline AS2 & & & & & \\
\hline AS3 & & & & & \\
\hline AS4 & & & & & \\
\hline AS5 & & & & & \\
\hline
\end{tabular}

Lo que les sucede a las personas transgénero en las escuelas señala Berkins, (2007) es que se topan con una serie de mecanismos que luego generan el miedo a la discriminación y que las impiden seguir estudiando. "La escuela se vive como un espacio en el que somos agredidas por docentes y compañeros y compañeras, es uno de los ámbitos donde más violencia se vive" (p. 72)

Me sentí ridiculizado y burlado cuando uno de mis maestros empezó a criticar mi forma de hablar y mover mis manos en una exposición, y sin darme cuenta todo el salón estaba en lo mismo, tomé mis libros y salí corriendo del salón, llore mucho, llegue a sentirme muy solo... es 
muy difícil enfrentar todos los días lo mismo hasta que llegó momento y no asistí más. (Actor Social 5).

El contexto escolar se ha convertido en un espacio de discriminación y violencia, impidiendo que los niños y los jóvenes disfruten de su derecho fundamental a una educación de calidad (UNESCO,2017).

Una vez salí del salón a tomar agua y un grupo de compañeros me agarraron en el baño y me golpearon, me gritaban cosas, me tomaron a la fuerza y me pintaron mi cara con lápiz. labial... Lloré mucho, desde ese día no quise volver a la escuela eso fue uno de los motivos que me hizo abandonar los estudios (Actor Social 1).

Las circunstancias hostiles que marcan la experiencia de escolarización de la mayoría de las niñas y adolescentes transgenero condicionan severamente las posibilidades en términos de inclusión social y de acceso a un empleo de calidad en la adultez" (Berkinns, 2007 p.67)

(...) ese día llegue al colegio como siempre temprano, y prácticamente el director me estaba esperando, me llevo a su oficina y me dijo que si no cambiaba mi forma de ser y no me comportaba como un niño más no podría seguir en la institución, me dijo que mi comportamiento dañaba la imagen del colegio. (Actor Social 3).

En virtud de esta experiencia Barriro (2016) expone que lo que sucede a menudo es también que la escuela no excluye (discursivamente) pero condiciona la inclusión, siempre que nieguen su identidad al ingresar a la escuela lo pueden hacer, ejemplo: venir vestida/o según su sexo biológico.

\section{Cuarto concepto emergente: Contexto Laboral}

En el contexto laboral las personas trans están expuestas a mayores riesgos dado que durante décadas, el mercado laboral ha estado prácticamente cerrado para ellas, provocando hayan terminado en una situación de extrema exclusión social, (Federación Estatal de Lesbianas, Gais, Transexuales y Bisexuales, 2017) Para otras personas, la necesidad de mantener un trabajo les ha obligado a proteger su identidad, y aquellos que se han atrevido a darla a conocer de manera natural han tenido que sufrir problemas de todo tipo que van desde el acoso laboral, la extorsión, el cambio de categoría profesional o incluso el despido. 


\section{Cuadro 4}

Acciones de violencia dentro del factor Laboral

\begin{tabular}{|c|c|c|c|c|c|}
\hline \multicolumn{6}{|c|}{ Factor Laboral } \\
\hline & \multicolumn{5}{|c|}{ Acciones } \\
\hline Actor social & Discriminación & Exclusión & Restricción & Rechazo & Despido \\
\hline \multicolumn{6}{|l|}{ AS1 } \\
\hline \multicolumn{6}{|l|}{$\mathrm{AS} 2$} \\
\hline \multicolumn{6}{|l|}{ AS3 } \\
\hline \multicolumn{6}{|l|}{ AS4 } \\
\hline AS5 & & & & & \\
\hline
\end{tabular}

El trabajo es un factor constitutivo de la vida misma, te genera tranquilidad, seguridad y calidad de vida. Situación que para la mayoría de las personas transgenero se convierte en un dilema dado a la discriminación exclusión, restricción, preferencia u otro trato diferente, los mismos se develan en las siguientes narrativas:

Mi mayor deseo es tener un trabajo fijo donde me gane la vida haciendo lo que me gusta, uno donde me valoren por lo que se hacer, hasta ahora no he tenido esa oportunidad, ahora trabajo de ayudante en una peluquería. (Actor Social 3).

Solo deseo tener un trabajo decente, donde me sienta bien, soy analista de sistema tengo muchos conocimientos solo quiero una oportunidad, siento frustración, rabia y tristeza de que en algunos lugares no nos acepten (Actor Social 5).

Tengo mi trabajo, pero debo fingir lo que realmente soy. me da mucho miedo que me despidan, además que ya he sufrido por las burlas y los rechazos no quiero que esto mismo pase en mi trabajo (Actor Social 1).

\section{Factores de Resiliencia}

\section{Modalidad de interacción en las categorías Yo soy, yo estoy}

Esta modalidad de interacción en la categoría yo soy, yo estoy alude a la fortaleza interna y a la autoimagen entre otros aspectos. En el transitar al proceso de resiliencia, los actores sociales a través de expresiones linguísticas han develado como ellos se han apropiado de su realidad, al comportamiento saludable y a superación de eventos traumáticos. 


\section{Cuadro 5}

Modalidad de interacción en la categoría Yo soy, Yo estoy

\begin{tabular}{|c|c|c|c|c|c|c|c|}
\hline \multicolumn{2}{|c|}{ Condiciones de base } & \multicolumn{2}{|c|}{ Visión de sí mismo } & \multicolumn{2}{|c|}{ Visión de problema } & \multicolumn{2}{|c|}{ Respuestas resilientes } \\
\hline$\overline{\text { F1 }}$ & Identidad & $\mathbf{F 2}$ & Autoestima & F3 & Satisfacción & F4 & Pragmatis mo \\
\hline \multicolumn{2}{|c|}{$\begin{array}{l}\text { Una persona que los } \\
\text { demás quieren }\end{array}$} & \multicolumn{2}{|c|}{$\begin{array}{l}\text { Una persona que los } \\
\text { demás respetan }\end{array}$} & \multicolumn{2}{|c|}{$\begin{array}{l}\text { Un modelo positivo } \\
\text { para otras persona }\end{array}$} & \multicolumn{2}{|c|}{ Una persona práctica } \\
\hline \multicolumn{2}{|c|}{$\begin{array}{l}\text { Una persona que le } \\
\text { gusta ayudar }\end{array}$} & \multicolumn{2}{|c|}{$\begin{array}{l}\text { Una persona que } \\
\text { despierta simpatía en } \\
\text { los otros }\end{array}$} & \multicolumn{2}{|c|}{$\begin{array}{l}\text { Yo estoy seguro de } \\
\text { mí mismo }\end{array}$} & \multicolumn{2}{|c|}{$\begin{array}{l}\text { Una persona con metas } \\
\text { en la vida }\end{array}$} \\
\hline \multicolumn{2}{|c|}{$\begin{array}{l}\text { Una persona que } \\
\text { demuestra afecto }\end{array}$} & & & & & \multicolumn{2}{|c|}{$\begin{array}{l}\text { Una persona que termina } \\
\text { lo que empieza }\end{array}$} \\
\hline
\end{tabular}

Es de acotar que para lograr este proceso de resiliencias los actores sociales comentan que el despertar y reconocerse como seres valiosos tiene como crédito la ayuda de grupos de apoyo personas que vivenciaron las mismas experiencias y los han encaminado todo el proceder hacia la resiliencia. Con base en lo que implica la categoría a "yo soy, yo estoy" se hace presenta la confianza como uno de los pilares básicos sobre el que descansan la motivación para enfrentar a los retos de la vida, la capacidad para establecer buenas relaciones con los demás y hacer autoafirmación ante ellos, asumir responsabilidades, aceptar los fracasos y un sinfín de aspectos que necesitan de la confianza propia para gestionarlos de manera óptima, (Montejano, 2018)

No fue fácil romper con los viejos conceptos que la sociedad me había imputado. yo me sentía nadie... inseguro de mí mismo.... veía a todas las personas como enemigos. Una vez que entro a los grupos de apoyo comienzo a darme cuenta de valor como persona y como ser humano que tengo, hoy tengo otras perspectivas de la vida, y con mucho deseo lograr mis metas (Actor Social 3)

Una de las cosas que siempre me han caracterizado es que me gusta brindar ayuda ... y yo no había tomado en cuenta mis fortalezas, soy una persona amorosa, respetuosa, y reconocer mis potencialidades me han ayuda a valorarme y quererme a mí mismo (Actor Social 1)

La autoestima de acuerdo a Espina (2019) no es un rasgo estático, y varía según cada persona. Dentro de la categoría "yo soy" "yo estoy" se pueden apreciar dos componentes: un sentimiento de capacidad personal y un sentimiento de valía personal, ambas reflejan la habilidad de la persona para enfrentarse y superar los problemas y para respetar y defender sus intereses y necesidades. 
Estoy más centrado en mí, y les restó importancia a los comentarios ajenos que no me aportan nada, reconozco lo valioso que soy y por estar pendiente de los demás y de las burlas me había olvidado de mí, hoy me reconozco, me aprecio tengo mis metas claras, he entendido que el amor empieza por uno mismo, y a través de estos detalles las personas han comenzados a respetarme. (Actor Social 4).

\section{Factores de Resiliencia: Modalidad de interacción en la categoría "Yo tengo"}

Esta categoría indica al apoyo que reciben las personas para encaminarse a la resiliencia, el amor de la familia de los amigos, en la que la orientación de profesionales es de suma importancia.

\section{Cuadro 6}

Modalidad de interacción en la categoría "Yo tengo"

\begin{tabular}{|c|c|c|c|}
\hline $\begin{array}{c}\text { Condiciones de } \\
\text { base }\end{array}$ & $\begin{array}{l}\text { Visión de si } \\
\text { mismo }\end{array}$ & Visión de problema & Respuestas resilientes \\
\hline \begin{tabular}{l|l} 
F5 & Vinculo \\
\end{tabular} & \begin{tabular}{l|l} 
F6 & Redes \\
\end{tabular} & \begin{tabular}{l|l} 
F7 & Modelo
\end{tabular} & \begin{tabular}{l|l} 
F8 & Metas \\
\end{tabular} \\
\hline $\begin{array}{l}\text { Relaciones } \\
\text { personales } \\
\text { confiables }\end{array}$ & $\begin{array}{l}\text { Personas que me } \\
\text { apoyan }\end{array}$ & $\begin{array}{l}\text { Un ambiente de trabajo o } \\
\text { estudio estable }\end{array}$ & Mis objetivos claros \\
\hline $\begin{array}{l}\text { Una familia bien } \\
\text { estructurada }\end{array}$ & $\begin{array}{l}\text { A quien recurrir en } \\
\text { caso de problemas }\end{array}$ & $\begin{array}{l}\text { Personas que me ponen } \\
\text { límites razonables }\end{array}$ & $\begin{array}{l}\text { Personas con quien enfrentar } \\
\text { los problemas }\end{array}$ \\
\hline $\begin{array}{l}\text { Relaciones } \\
\text { afectivas sólidas }\end{array}$ & $\begin{array}{l}\text { Personas que } \\
\text { estimulan mi } \\
\text { autonomía e } \\
\text { iniciativa }\end{array}$ & $\begin{array}{l}\text { Personas que me ayudan a } \\
\text { evitar peligros } \mathrm{o} \\
\text { problemas }\end{array}$ & Proyectos a futuro \\
\hline
\end{tabular}

emocional, para, Díaz y Sánchez-López (2002), los individuos que presentan sanas relaciones tienden a ser emprendedores y decisivos, respetan las normas propias de la sociedad; se muestran alegres y seguros den ámbito social.

Este caminar se me ha hecho más fácil desde que conocí a la persona que hoy vive conmigo, estos procesos son muy difíciles vivirlos en soledad, siempre se necesita alguien te escuche, te aconseje, te oriente, alguien que evite que te metas en problemas (Actor Social 5)

La redes de apoyo, cumplen como función desarrollar mecanismos que permitan ayudar a las personas que se encuentran en situaciones que no sean claras para su bienestar personal son las organizaciones sociales que buscan mecanismos de acción positiva para generar un espacio 
seguro ante los diferentes contextos de discriminación que tienen cada una de ellas (SolanoMarcillo, y Mera-Leones, 2020)

Desde que comencé a asistir a la red de apoyo enseguida comencé a ver cambios en mi vida, alli he conseguido personas en la que puedo confiar... retome mis estudios y voy por la vida con mayor seguridad... una cosa que entendi es que si quieres que te acepten como eres debes empezar por aceptarte a ti mismo no imponer lo que eres (Actor Social 2)

Comencé a estudiar de nuevo, y tengo un trabajo estable donde me respetan, eso me tiene la autoestima por el cielo... y gracias a la red de apoyo donde asisto me mantengo firme con mis proyectos de vida, me gusta asistir allí... me siento seguro de poder llorar $y$ desahogarme cuando lo necesito (Actor Social 3).

\section{Factores de Resiliencia: Modalidad de interacción en la categoría a "Yo puedo"}

Esta categoría hacer referencia a las posibilidades de expresión, búsqueda de ayuda, enfrentamiento de dificultades, capacidad de aprendizaje, entre otras, las personas entran en un estado de empoderamiento, de fuerza interior que les ayuda tener una visión distinta de los problemas y encontrar en ellos la posibilidad de ser creativo para resolverlos.

\section{Cuadro 7}

Modalidad de interacción en la categoría a "Yo puedo"

\begin{tabular}{|c|c|c|c|}
\hline $\begin{array}{c}\text { Condiciones de } \\
\text { base }\end{array}$ & $\begin{array}{l}\text { Visión de si } \\
\text { mismo }\end{array}$ & Visión de problema & Respuestas resilientes \\
\hline Afectividad & Autoeficacia & \begin{tabular}{l|l|} 
F11 & Aprendizaje \\
\end{tabular} & \begin{tabular}{l|l} 
F12 & Ge ratividad
\end{tabular} \\
\hline Tener buen humor & $\begin{array}{l}\text { Resolver problemas de } \\
\text { manera efectiva }\end{array}$ & Ser creativo & $\begin{array}{l}\text { Generar estrategias } \\
\text { para solucionar mis } \\
\text { problemas }\end{array}$ \\
\hline $\begin{array}{l}\text { Establecer } \\
\text { relaciones } \\
\text { personales de } \\
\text { confianza }\end{array}$ & $\begin{array}{l}\text { Equivocarme y luego } \\
\text { corregir mi error }\end{array}$ & Ser persistente & $\begin{array}{l}\text { Esforzarme por lograr } \\
\text { mis objetivos }\end{array}$ \\
\hline $\begin{array}{l}\text { Hablar de mis } \\
\text { emociones }\end{array}$ & $\begin{array}{l}\text { Apoyar a otros que } \\
\text { tienen dificultades" }\end{array}$ & $\begin{array}{l}\text { Aprender de mis } \\
\text { aciertos y errores }\end{array}$ & $\begin{array}{l}\text { Trabajar con otros en la } \\
\text { solución de problemas }\end{array}$ \\
\hline
\end{tabular}

La Autoconfianza tiende a ser un proceso intelectual en el cual las personas tienen seguridad en sí misma que dominan una situación, la sensación y el convencimiento de que es competente, y valora todas sus posibilidades. 
Asimismo, dentro de la categoría "yo puedo" se desataca el humor como un aspecto importante de la resiliencia. En este sentido Gutman (2018) señala que una de las principales características de las personas resilientes es su sentido del humor, Alguien que sabe reírse de sí mismo, que hace bromas sobre la adversidad, tiene mucha más facilidad para superar los problemas que alguien que carece de sentido del humor.

Enfrentarse con humor a la adversidad no quiere decir otra cosa que aceptar su parte positiva, o encontrar su lado absurdo, o descubrir lo que tiene de extraordinario y utilizarlo para hacernos sentir mejor, para reír. No se trata, por tanto, de negar el problema, sino de afrontarlo con una actitud diferente, positiva.

Yo me había olvidado de reír, de soñar, para mi estar vivo era un problema, cuando comienzo a quererme y aceptarme tal como soy, empecé a ver la vida diferente, tengo buen humor, aprendí a reírme de mi mismo. (Actor Social 1)

Una de las cosas que he aprendido en todo este proceso ha sido verbalizar mis emociones, sin miedo a ser criticado, expresar lo que me pasa, lo que siento, lo que me agrada, y lo que no, además que te hace sentir bien uno siente que se quita un peso de encima, además comienzas tener confianza en las demás personas (Actor Social 4)

Toda esta experiencia ha sido un gran aprendizaje en mi vida, y con base a ello he comenzado a ayudar a otras personas... estoy estudiando en una universidad online, y he podido hablar abiertamente de quien soy... me estoy preparando, tengo entre mis proyectos de vida escribir un libro no para que conozcan que soy una persona transgenero, sino para que mis experiencias ayuden a otros y a otras a valorarse. (Actor Social 2)

La resiliencia es un proceso que se experimenta y se vive en la que se pone de manifiesto el malestar, el estrés, además que exigen hacer esfuerzos emocionales, consciente para cambiar las formas de percibir, pensar y actuar de la realidad desde una situación adversa y vulnerable, por lo tanto, genera aprendizaje, no solo de la situación que se vive, sino de conocerse a sí mismo y valorarse desde la propia existencia, incrementado la autoestima y la autoconfianza para enfrentar nuevos nuevas experiencias de éxito y fracasos.

\section{Reflexiones Finales}

En función de los resultado generados en este estudio es oportuno exponer las siguientes reflexiones: La escuela, la familia y sociedad son instituciones donde el ser humano se 
desarrolla crece, tiene expectativas y hace vida, por lo tanto es deber de estas instituciones abrir el compás de todas las posibilidades que permitan la inclusión y la no exclusión de las personas transgenero, dado que su condición e identidad de vida no resta a su condición de humano, y como todo ser humano se le deben respetar y proteger sus derechos y dignidad.

Se denota claramente que los jóvenes transgénero que expresan su identidad suelen ser castigados por transgredir las normas impuestas por el sistema social, cultural y religioso, llevándolos a vivir situaciones de vulnerabilidad. En esta sociedad moderna se amerita con urgencia generar cambios estructurales en la familia, en la educación y en ambientes sociales, que permitan el reconocimiento y la igualdad sin discriminación a las personas con identidad transgenero, generándoles oportunidades de vida para estudiar, trabajar y gozar de la compañía y cuidado de una familia.

Asimismo, se debe detener la constante discriminación y estigmatización en los diferentes entornos (social, familiar y educativo) para evitar el deterioro de la salud emocional y psicológica de quienes viven esas experiencias, y con ello protegerlos de sintomatología de depresión, ansiedad, suicidio y otro tipo de conductas riesgosas como consumo de sustancia y prostitución.

Con base a lo expresado es imprescindible generar estrategias educativas y sociales, que permitan organizar procesos de intervención psicológica a través de redes de ayuda con grupos especializados, que permitan brindar espacios de equidad y de interacción social para desarrollar en los jóvenes transgenero procesos de resiliencias. Crear espacios de apoyo a través de la educación para hacer de esta un lugar sin distinción en donde todos tenga cabida dentro de un ambientes transformador e igualitario.

\section{Referencias}

Apiarkorvski, F. (2012). Vulnerabilidad de derechos en personas trans. IV Congreso Internacional de Investigación y Práctica Profesional en Psicología XIX Jornadas de Investigación VIII Encuentro de Investigadores en Psicología del MERCOSUR. Facultad de Psicología Universidad de Buenos Aires, Buenos Aires. https://www.aacademica.org/000-072/55.pdf.

Alarcón de Soler, M. (2006). La perspectiva vincular en psicoanálisis Aportes de la Escuela de Isidoro Berenstein y Janine Puget en el abordaje de los vínculos. 
Presentación Sociedad Colombiana de Psicoanálisis, 1 - $11 .$. http://bibliotecadigital.usbcali.edu.co/bitstream/10819/3377/3/Influencia_Familia_Cons olidacion_Jaramillo_2015.pdf.

Bareiro, M. (2016). La exclusión de las personas trans del sistema educativo: un análisis de la experiencia en la educación de personas jóvenes y adultas en Paraguay. http://biblioteca.clacso.edu.ar/clacso/becas/20160401010040/informefinaldeinvestigacion laurabareiro.pdf

Berkins, Lohana (comp.) 2007. Cumbia, copeteo y lágrimas (Buenos Aires: Editorial A.L.I.T.T. - Asociación de lucha por la identidad Travesti- Transexual)

Borella,Y. Campillay, M. Gómez, J. Verón, C. y Zalazar. E.(2019) Discriminación laboral a

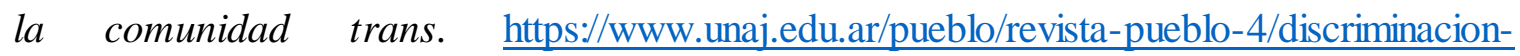
laboral-a-la-comunidad-trans/

Budge, S. L., Adelson, J. L., \& Howard, K. S. (2013). Anxiety and depression in transgender individuals: the roles of transition status, loss, social support, and coping. Journal of Consulting and Clinical Psychology, 81(3), 545-557. doi: 10.1037/a0031774

Cardoso, M. (2019). Estudio de la vulnerabilidad y la resiliencia en la ciudad de Santa Fe, Argentina: El rol de los servicios urbanos en general y del transporte de pasajeros en particular. Revista de geografía Norte Grande, (73), 133159. https://dx.doi.org/10.4067/S0718-34022019000200133

Cifuentes, R. (2011). Diseño de proyectos de investigación cualitativa. Buenos Aires. Argentina: Noveduc.

Díaz Barriga, F, \& Reséndiz Morán, A (2017). Factores de resiliencia y vulnerabilidad en jóvenes afectados por la violencia en Ciudad Juárez, Chihuahua. Argumentos, 30(84),147-168.: https://www.redalyc.org/articulo.oa?id=59552650008

Díaz, J. F. \& Sánchez-López, M. P. (2002). Relaciones entre estilos de personalidad y satisfacción autopercibida en diferentes áreas vitales. Psicothema, http://repositorio.ual.es/bitstream/handle/10835/2298/Trabajo.pdf?s equence=1

Espina, M. (2019) la autoestima: valoración de uno mismo. https://marisaespina.com/laautoestima-y-valoracion-de-uno- mismo/

Fernández, O. (2007). Transexualidad, homosexualidad y familia (reflexiones teóricas y resultados de trabajo desde una visión de la psicología). Revista del Instituto de 


Ciencias Jurídicas de Puebla $\quad$ A.C, $26-41$.
http://www.redalyc.org/pdf/2932/293222932002.pdf

González, F. (2006). Investigación cualitativa y subjetividad. Oficina de Derechos Humanos del Arzobispado de Guatemala.

Granado, L. (2020) Resiliencias en personas transgenero. https://nkineuropsicologia.com/resiliencia-en-personas-transgenero/

Grotberg, E. (2009) La resiliencia en el mundo de hoy: Como superar la adversidad. GEDISA. España. https://www.pnas.org/content/100/14/8074.

Gutiérrez M., Cacao, S. y Ormaza M.(2017): La inserción laboral de los trangéneros en la cuidad de Portoviejo 2017", Revista Contribuciones a las Ciencias Sociales, https://www.eumed.net/rev/cccss/2017/03/insercion-laboral-trangeneros.html

Gutman. R. (2018) El poder oculto de la sonrisa . https://psicoasistencia.com/resiliencia-ysentido-del-humor/

Hoffman, B. (2014). An overview of depression among transgender women. Depression Research and Treatmen, 1-9. doi:http://dx.doi.org/10.1155/2014/394283

Kotliarenco, M.Cáceres, I. y Álvarez, C. (1996). Resiliencia. Construyendo en adversidad. Santiago de Chile: CEANIM.

Montejano, S. (2018) La confianza en uno mismo: las claves. https://www.psicoglobal.com/blog/confianza-en-uno-mismo

Perezi, A. (2016). La importancia del apoyo familiar (y social) en el desarrollo psicológico de niños y niñas transgénero. Aproximaciones desde una perspectiva de derechos humanos. https://dpicuantico.com/sitio/wp-content/uploads/2016/07/Columna-de-Opinion1-

SDCBYDH-Nro-14-04.01.pdf

Piñero, M, Rivero, M, Riera, E. (2019) Proceder del Investigador cualitativo: Precisiones para el proceso de investigación Editor UPEL-IPB FONDEIN UPEL Barquisimeto, Venezuela.

Yogyakarta (2007). Principios sobre la aplicación De la legislación internacional de derechos humanos en relación con la orientación sexual y la identidad de género.yogyakartaprinciples.org

Rohner, Ronald P., \& Carrasco, Miguel Á. (2014). Teoría de la Aceptación-Rechazo Interpersonal (IPARTheory): bases conceptuales, método y evidencia 
empíricaInterpersonal Acceptance-Rejection Theory (IPARTheory): theoretical bases, method and empirical evidence. Acción Psicológica, 11(2), 9-26. https://dx.doi.org/10.5944/ap.11.2.14172

Ruiz Cortes, E. (2017) Depresión y apoyo familiar en personas transgénerohttps $/ /$ repository.urosario.edu.co/bitstream/handle/10336/13484/Tesis\%20fin al\%20de\%20Tatiana\%20Ruiz.pdf? sequenc.

Solano-Marcillo, K. Mera-Leones R. (2020) Redes de apoyo y resilencia en mujeres transgéneros: caso ciudad de bolívar, Ecuador. https://doi.org/10.46296/yc.v4i7.0051

Strauss, A. y Corbin J. (2002). Bases de la investigación cualitativa. Técnicas y procedimientos para desarrollar la teoría fundamentada. Colombia: Editorial Universidad de Antioquia.

Taylor,S.J. Bogdan, R (1992).-Introducción a los métodos cualitativos en investigación. La búsqueda de los significados. Editorial. Paidós, España.

UNESCO (2017) Violencia escolar.https://www.unfe.org/wp-content/uploads/2017/05/Bullyingand-Violence-in-School-Esp.pdf

UNESCO (2019) La violencia homofóbica y transfóbica en la escuela es un problema mundial, según un informe. https $/ /$ es.unesco.org/news/violencia-homofobica-y-transfobicaescuela-es-problema-mundial-segun-informe 